\title{
Time dependence of stimulation/recording-artifact transfer function estimates for neural interface systems
}

\author{
Nick Chernyy, Student Member, IEEE, Steven J. Schiff, Bruce J. Gluckman, Member, IEEE
}

\begin{abstract}
-
A continuous feedback-enabled control system requires simultaneous measurements of the system states and generation of a control output. In neural systems, electric stimulation used to interact with neural activity also creates additional electrical potential variations at measurement points used to monitor neural activity. This stimulus artifact confounds recording of underlying neural activity through the addition of both common mode and differential potentials. We model this artifact as a linearly filtered version of the applied electrical current. We demonstrate a method to determine the properties of this filter using multi-taper techniques for chronically implanted animals stimulated with polarizing low-frequency electric fields (PLEF). When measured repeatedly in chronic experiments with continuous recordings, we observe slow changes of up to $50 \%$ transfer function magnitude (figure 1) . Such changes reflect a combination bulk impedance changes of the tissue and changes in electrode interface properties. These variations
\end{abstract}

Nick Chernyy at the Engineering Science and Mechanics Department, The Pennsylvania State University, State College, PA 16802 USA; e-email: nchernyy@psu.edu.

Steven J. Schiff is at the Department of Neurosurgery and Department of Engineering Science and Mechanics, The Pennsylvania State University, University Park, PA.

Bruce J. Gluckman is at the Department of Engineering Science and Mechanics, and Department of Neurosurgery, The Pennsylvania State University, University Park, PA. (phone: 814865-0178; e-mail: bjg18@psu.edu).

This work has been supported by the National Institutes of Health through grants R01EB001507, R01MH50006 and K02MH01493. This project was funded, in part, with grants from the Pennsylvania Department of Community and Economic Development Keystone Innovation Zone Program, and the Pennsylvania Department of Health using Tobacco Settlement Funds (the Departments specifically disclaim responsibility for any analyses, interpretations or conclusions). need to be tracked and accommodated for successful chronic continuous feedback neural control systems.

Index Terms-neural recording, neural stimulation, artifact subtraction, epilepsy, feedback control, seizures, transfer function, multi-taper, DPSS

\section{INTRODUCTION}

$\mathbf{M}$ $\mathrm{UCH}$ progress has been made over the past century in applications of electrical stimulation to the central nervous system for treatment of neurological disorders and restoration physical function. Many of the systems developed cannot perform concurrent stimulation and recording and therefore lack the bandwidth required for continuous feedback control. This problem arises when stimulation artifact, caused by the flow of stimulation current through conductive neural tissue, is recorded along with electrophysiological signals used to monitor neural activity and thereby confounds the observation of the neural network state. Typical solutions have been to perform recordings far away from the electrical stimulation site, or to ignore recorded data that is corrupted by artifact. These solutions separate the analyzed data from the stimulus spatially or temporally. Neither of these solutions can therefore take full advantage of continuous feedback control. We demonstrate here, in the context of polarizing low-frequency electrical field (PLEF) neural modulation, an efficient method for artifact removal applicable to arbitrary stimulus waveforms. This enables us to apply PLEF modulation for real-time feedback applications. 
We proposed [1] a linear impedance model 1 which can be derived from lead-field theory [2] [3] or electromagnetic field derivations in conductive media [4]. The key then to performing artifact subtraction is the computation of the linear relationship between the stimulus current and the additive electrical potential between a pair of recording electrode sites in the presence of ongoing neural activity. It is additionally important to sense slow changes in transfer function that would reflect changes in bulk tissue impedance or electrode configuration.

$$
V_{\text {recorded }}(f)=T(f) \cdot I_{\text {stimulus }}(f)+\eta(f)
$$

We've presented this model in the frequency domain. The transfer function $T(f)$ is the complex, linear relationship between the applied stimulus current $I_{\text {stimulus }}$ and the recorded physiological potential $V_{\text {recorded }}$ with $\eta$ encompassing background neural activity and any non-linear effects. We demonstrate an efficient method for the computation of $T(f)$ which can typically be converted into a stable and causal digital filter for real-time artifact subtraction. Furthermore, these methods are robust against non-linear effects [5] and provide estimates of the first two statistical moments [6] which can be used in hypothesis testing to determine artifact subtraction efficacy.

\section{METHODS}

$\mathbf{T}$ HE the methods presented here act as a logical continuation of our previous work [1]. We use a specially-crafted stimulus waveform to determine the best possible fit for $T(f)$ in equation 1 using a linear regression and design a digital filter to match the transfer function and predict the stimulus artifact given an arbitrary stimulus current waveform.

We perform frequency-domain computations based on time-domain data. Therefore, careful selection of data-tapers must be made to minimize bias and variance of our Fourier estimates [7]. Spectral estimator bias and variance are reduced through the use of multiple discrete prolate spheroidal sequences (DPSS), or Slepian tapers [8] [6], at expense of being unable to distinguish close spectral components that are within one taper bandwidth
$W=j /(N \Delta t)$ of each other (p.335 [7]). These tapers are orthogonal, so that each taper yields an independent Fourier estimate, with a consistent phase, for the same time window of a signal. If the signal results from a noise process, then the orthogonal samples will be independent samples of the noise process, which, in the frequency domain will appear with random phase but, on average, the same power. This forms the basis for Thomson's harmonic analysis to detect lines in spectra over noise [6], and the basis of our extraction technique as follows.

First, we apply a probe current, (equation 2), formed from sinusoidal components that are coherent for times longer than a window period. Then we use the multi-taper Fourier methods to estimate the line (sinusoidal components appear ideally as delta functions, or lines, in Fourier spectra) amplitudes and variance estimates for both the applied current and the combination of artifact, which will be composed of the same sinusoidal frequencies, plus the neural recordings. We then assume the neural component $\eta$ is primarily incoherent across time windows and orthogonal samples. Finally, we then use a weighted least squares analysis to determine the optimal $T(f)$.

$$
I_{\text {stim }}(t)=\sum_{k=1}^{p} a_{k} \sin \left(2 \pi f_{k}+\phi_{k}\right) ; \quad \phi_{k} \in(-\pi, \pi)
$$

The bandwidth limitations of the multi-taper method require that the sinusoids which make up the stimulus waveform are at frequencies $\left(f_{k}\right)$ that are $>2 W$ apart and that sums of their frequencies are also $>2 W$ apart to avoid quadratic effects [5]. Higher-order combinations will have lower power and will therefore contribute much less to any analysis performed. Furthermore, randomization of the initial phases $\left(\phi_{k}\right)$ for each frequency component can give our interrogatory stimulus waveform similar statistical properties to white noise over the frequency range of interest. This careful stimulus signal construction for the purpose of minimizing spectral leakage is a key advantage over other transfer function estimation methods that rely on filtering a white noise sources which results in a 


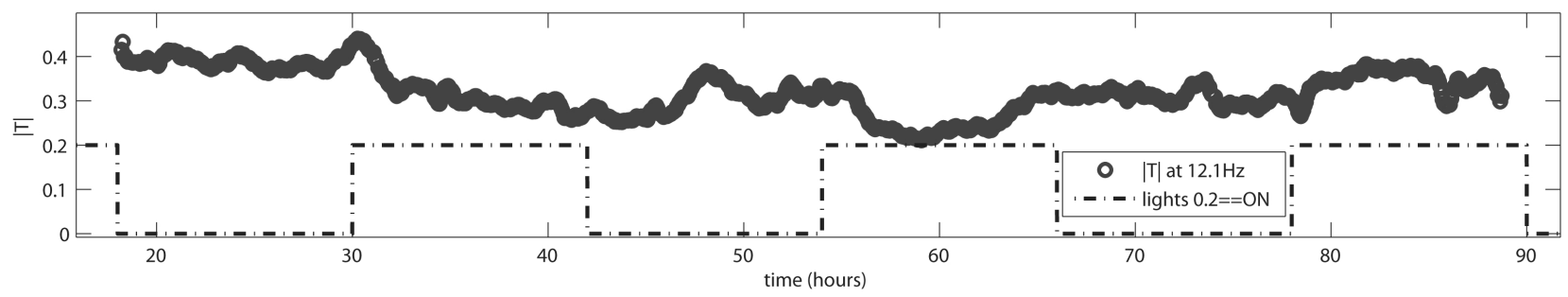

Fig. 1. Example transfer function amplitude at $12.7 \mathrm{~Hz}$ as a function of time in a single animal. Transfer functions were estimated every $400 \mathrm{~s}$. Imposed room lights are plotted to indicate day/night cycle (rats sleep in the light cycle).

uniform power spectrum over the frequency band of interest and therefore suffer from spectral leakage. The interrogation waveform $I_{\text {stim }}$ used to estimate the transfer function and is composed of $p$ distinct sinusoids so as to span the frequency range of typical stimulus waveforms whose artifacts will be subtracted. The amplitudes $a_{k}$ are uniformly distributed so that the overall stimulus power fits a prescribed power specification.

We use data from several adjacent presentations of the stimulus waveform to compute vectors $\bar{V}_{r}(f)$ and $\bar{I}_{s}(f)$ which lead to an optimal weighted leastsquares estimate of $T(f)$ in equation 1 at the frequencies which make up the stimulus waveform. This operation defines the transfer function response as a set of complex values at the stimulated frequencies and can be used to realize a causal digital filter. The causality of this filter realization will permit its use to predict the stimulus artifact by applying the matched $T(f)$ filter to the stimulus current waveform in real-time, either in the digital or analog domain, and predicting the stimulus artifact, of an arbitrary waveform within the frequency range of interest, for subtraction.

The ability to accurately estimate the transfer function and thereby accurately perform artifact subtraction becomes a signal-to-noise ratio issue and is mainly dependent on the power ratio of the stimulus artifact (pre-subtraction) and the background neural activity. Substantial simulations demonstrate typical residual artifact, after artifact subtraction, to initial artifact power ratios of $-69 \mathrm{~dB}$, $-49 \mathrm{~dB}$, and $-24 \mathrm{~dB}$ when the initial stimulus artifact signal to noise rations were $20 \mathrm{~dB}, 0 \mathrm{~dB}$, and $-20 \mathrm{~dB}$ respectively.

\section{EXPERIMENTAL PROCEDURE}

$\mathbf{M}$ ULTIPLE Sprague-Dolly rats (male, 350400g, $N>10$ ) were each implanted with a pair iridium-oxide coated stainless-steel stimulating electrodes $\left(\approx 3 \mathrm{~mm}^{2}\right.$ surface area $)$ in compliance with IACUC-approved experimental protocols. Three $125 \mu \mathrm{m}$ bipolar electrode pairs were implanted in the hippocampi and two pairs of cortical screw electrodes were inserted into the skull. After adequate recovery, each animal was connected to a chronic recording and stimulation system designed for PLEF stimulation [9]. Probe stimuli for computing transfer functions, along with example stimuli waveforms, were applied intermittently or periodically to assess time dependence and fidelity of transfer function estimation in the range $0.5-35 \mathrm{~Hz}$ range. Data from bipolar recordings with unstable or unphysiological local-field potential recordings, (approximately $20 \%$ of the recorded channels) was excluded from analysis.

\section{RESULTS}

$\mathbf{E}$ XAMPLES of in vivo signals before and after artifact subtraction are shown in (figure 2) for both sinusoidal (left) and square wave (right) stimuli. These signals are not the ones used for transfer function estimation. Note that even the sharp structures of the square waves stimulus artifact are eliminated.

We have been able to reliably subtract stimulus artifact in all of the animals in this study that had reliable recording channels $(N>10)$, both from bipolar electrodes implanted in the hippocampus and differential screw electrode pairs over the cortex. 
Interestingly, we observed in these experiments a clear slow variation in transfer function amplitude on an hourly time scale, with a time course that seems correlated with diurnal cycle. Typical amplitude variations can be as much as $50 \%$, and were reliably observed in all four animals whose transfer functions for all recording channels were estimated at either one-hour or five-minute intervals over several days. An example time course in transfer function amplitude for $12.7 \mathrm{~Hz}$ is shown in figure 1 . Included in the figure is the imposed room illumination which is on a $12 / 12$ cycle. Note that the animal's sleep period is during the light phase.

Tri-axial animal head acceleration is continuously recorded in our experimental paradigm and did not demonstrate any statistically significant correlation with the transfer function magnitude other the expected increase in animal movement while the lights were off and decreased movement when the lights were on.

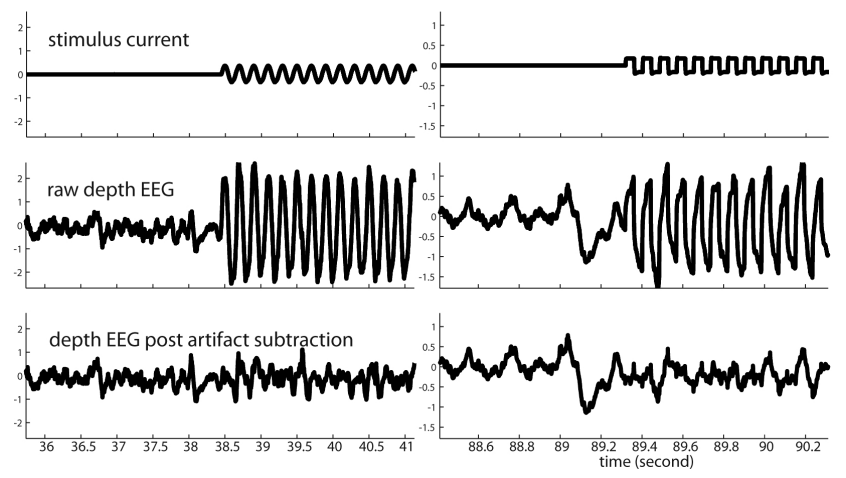

Fig. 2. Examples of artifact subtraction from a single animal during non-probe sinusoid (left) and square (right) stimulus current waveforms. The top traces are the stimulus current waveform, the middle traces are the raw depth recordings from a bipolar electrode. The bottom traces are the EEG after artifact subtraction.

\section{DISCUSSION}

W E have developed a method for estimating recorded artifact from electrical stimulation, and applied it in the case of chronically implanted animals stimulated with PLEFs. The artifact is accurately described by a linear transfer function, or filtered version, of the stimulation current.

Importantly, the magnitude of the transfer function slowly changes by as much as $50 \%$, with a nearly daily cycle. The tracking of such a timedependent transfer function is critical for real-time removal of stimulation artifact and implementation of real-time continuous feedback stimulation and control. We are currently working to modify our transfer function estimation algorithm to adaptively estimate transfer function based on its previous estimate and minimal probe current amplitude using Kalman-filter techniques.

The underlying conduction model used to justify the transfer function estimation, and equation 1 is strongly dependent on the geometric distribution of current between the stimulation electrodes and the detailed tissue impedance. Time variations in the transfer function can therefore be interpreted to arise from either changes in electrode configuration, which is unlikely, or from time-variations in tissue impedance. We are currently working to differentiate these.

\section{ACKNOWLEDGMENTS}

$\mathbf{T}$ HE authors would like to thank Dr. Sridhar Sunderam for helpful discussions and Dr. Giorgio La Corte and Madineh Sarvestani for their assistance with experiments.

\section{REFERENCES}

[1] Chernyy N., Schiff S. J., and Gluckman B. J. Multi-taper transfer function estimation for stimulation artifact removal from neural recordings. Conf Proc IEEE Eng Med Biol Soc, pages 2772-2776, 2008.

[2] Plonsey R. and Barr R. Bioelectricity: a quantitative approach. Springer, 3rd edition, 2007.

[3] Nunez P. and Srinivasan R. Electric fields of the brain: the neurophysics of EEG. Oxford University Press, 2005.

[4] Jackson J. D. Classical electrodynamics. Wiley, 3rd edition, 1998.

[5] Victor J. D. and Shapley R. A method of nonlinear analysis in the frequency domain. Biophysical Journal, 29:459-483, 1980.

[6] Thomson D. J. Spectrum estimation and harmonic analysis. Proceedings of the IEEE, 70(9):1055-1096, 1982.

[7] Percival D. B. and Walden A. T. Spectral analysis for physical applications. Cambridge University Press, 1993.

[8] Slepian D. Prolate spheroidal wave functions, fourier analysis, and uncertainty - v: the discrete case. The Bell Systems Technical Journal, 57(5):1371-1430, 1978.

[9] Sunderam S., Chernyy N., Peixoto N., Mason J. P., Weinstein S. L., Schiff S. J., and Gluckman B. J. Seizure entrainment with polarizing low frequency electric fields in a chronic animal epilepsy model. Journal of Neural Engineering, in press, 2009. 